PAPER

Structural, magnetic, magnetocaloric and specific heat investigations on $\mathrm{Mn}$ doped $\mathrm{PrCrO}_{3}$ orthochromites

To cite this article: Surendra Kumar et al 2017 J. Phys.: Condens. Matter 29195802

View the article online for updates and enhancements.
Related content

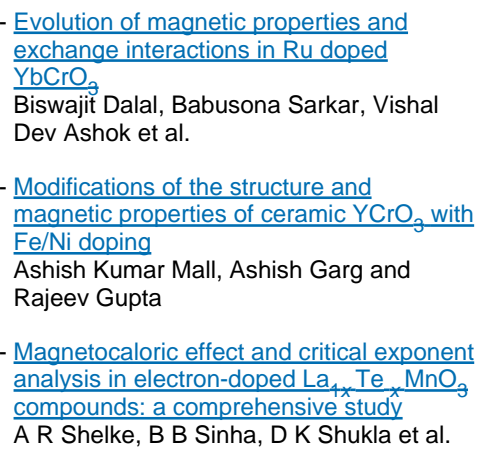

\section{Recent citations}

- $\frac{\text { Structural, Optical and Dielectric }}{\text { Properties of Bi Substituted Polycrystalline }}$
$\frac{\text { Praseodymium Chromate }}{\text { Ravikiran Late et al }}$
- Oxvgen vacancy modulating inverse and
$\frac{\text { conventional magnetocaloric effects }}{\text { coexisting in double perovskite }}$
$\frac{\text { Bi2NiMnO6- films }}{\text { Yulong Bai et al }}$
- A comparative study of the structural
$\frac{\text { optical, magnetic and magnetocaloric }}{\text { properties of HoCrO3 and }}$
$\frac{\text { HoCr0.85Cr0.15MnO3 orthochromites }}{\text { Komal Kanwar et al }}$




\title{
Structural, magnetic, magnetocaloric and specific heat investigations on Mn doped $\mathrm{PrCrO}_{3}$ orthochromites
}

\author{
Surendra Kumar ${ }^{1}$, Indrani Coondoo ${ }^{2}$, M Vasundhara ${ }^{3}$, Sandeep Kumar ${ }^{1}$, \\ A L Kholkin ${ }^{2,4}$ and Neeraj Panwar ${ }^{1}$ \\ ${ }^{1}$ Department of Physics, Central University of Rajasthan, Bandarsindri 305817, Ajmer, Rajasthan, India \\ 2 Department of Physics and CICECO-Aveiro Institute of Materials, University of Aveiro, 3810-193 \\ Aveiro, Portugal \\ 3 Materials Science and Technology Division, CSIR-National Institute for Interdisciplinary Science \\ and Technology, Industrial Estate, Trivandrum 695019, India \\ ${ }^{4}$ School of Natural Sciences and Mathematics, Ural Federal University, 620000 Ekaterinburg, Russia \\ E-mail: neerajpanwar@curaj.ac.in and neeraj.panwar@gmail.com
}

Received 8 January 2017, revised 3 March 2017

Accepted for publication 13 March 2017

Published 10 April 2017

\begin{abstract}
We have synthesized $\operatorname{PrCr}_{0.85} \mathrm{Mn}_{0.15} \mathrm{O}_{3}$ (PCMO) chromite and investigated the influence of manganese (Mn) doping at Cr-sites on the structural, magnetic, magnetocaloric and thermal properties of $\mathrm{PrCrO}_{3}$ compound. No structural transition was observed with Mn substitution and the doped compound crystallized into distorted orthorhombic structure with Pnma space group which was confirmed by Rietveld refinement of x-ray powder diffraction patterns. Neel temperature, noticed at $168 \mathrm{~K}$ from the temperature variation of magnetization, smaller than that reported for $\mathrm{PrCrO}_{3}$, indicated the influence of $\mathrm{Mn}^{3+}$ substitution in decreasing the antiferromagnetic ordering. Magnetization was almost eight times higher than that reported for undoped sample. Magnetocaloric effect measured via the magnetic entropy change and relative cooling power demonstrated significant values in the temperature range $10-20 \mathrm{~K}$. The values of magnetic entropy change are much superior to that reported for other polycrystalline orthochromites and even at smaller applied field strength. The material exhibited second order magnetic phase transition. The Debye temperature and the density of states at Fermi level were also calculated. The overall results make $\operatorname{PrCr}_{0.85} \mathrm{Mn}_{0.15} \mathrm{O}_{3}$ chromite a potential candidate to replace the existing materials for low temperature magnetic refrigeration.
\end{abstract}

Keywords: orthochromites, magnetic refrigeration, specific heat

(Some figures may appear in colour only in the online journal)

\section{Introduction}

Perovskite orthochromites are very promising materials for thermomagnetic switches, spintronic and thermally assisted magnetic random access memory (TAMRAM) devices [1-4]. Their general chemical formula is $R \mathrm{CrO}_{3}(R=$ rare earth or yttrium ion) having distorted orthorhombic structure (Pnma space group) and they exhibit canted antiferromagnetism in the G-type configuration below the Neel temperature $\left(T_{\mathrm{N}}\right)$ in the range $112-282 \mathrm{~K}$ which depends upon the ionic size of the tri-valent rare-earth ion present [5-10]. They also show the temperature induced magnetization reversal (i.e. the magnetization changes its polarity from positive to negative at one particular temperature known as compensation temperature) and spin reorientation transition at low temperatures (below $50 \mathrm{~K})[3,4,8,11]$. Below the Neel temperature, the spins of nearest $\mathrm{Cr}^{3+}$ ions do not possess the perfect antiparallel configuration, therefore, net magnetization is not zero and along with antiferromagnetic behaviour one observes weak ferromagnetic component also. This is known as canted antiferromagnetic 

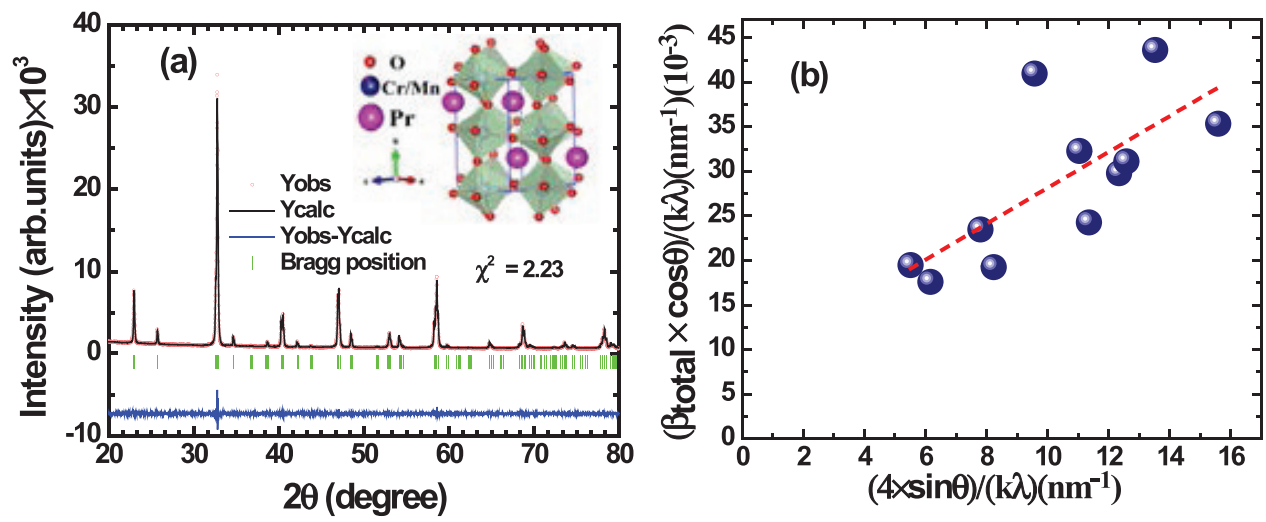

Figure 1. (a) The powder XRD profiles of PCMO showing the observed (red circle), calculated (black line), and difference intensities (blue line) along with the Bragg positions (green bars), inset shows the crystal structure of PCMO, (b) linear fit using the Williamson-Hall relation given by equation (1).

(CAFM) behaviour. Further, the $\mathrm{Cr}^{3+}$ ions produce the internal field at the rare-earth site [3, 6, 11]. Therefore, depending upon whether a magnetic ion, or non-magnetic ion is present at the rare-earth site, the internal field will (or will not) rotate the magnetic moment of the rare-earth ion in the opposite direction to that of $\mathrm{Cr}^{3+}$ ions. This way, the difference of the two magnetic moments will decide whether the particular orthochromite will demonstrate the magnetization reversal under low value of applied magnetic field. Whenever the applied magnetic field becomes greater than the internal field produced by chromium ions, the magnetization flipping along the field direction takes place resulting in the positive magnetization over the measurement range of temperature [4]. So far, many orthochromites like $\mathrm{YCrO}_{3}$ [12-14], $\mathrm{LaCrO}_{3}$ [15], $\mathrm{CeCrO}_{3}[3,16]$, $\mathrm{PrCrO}_{3}$ [17-19], $\mathrm{NdCrO}_{3}$ [20, 21], $\mathrm{SmCrO}_{3}$ [22-24], $\mathrm{GdCrO}_{3}$ $[25,26], \mathrm{DyCrO}_{3}[27,28], \mathrm{YbCrO}_{3}[4,29]$ and $\mathrm{HoCrO}_{3}[30$, $31]$ have been investigated for their complex magnetic properties and interesting results have been reported on them. As far as the $\mathrm{PrCrO}_{3}$ (PCO) orthochromite is concerned, only a very few studies exist on it [17-19]. For example, Gordon et al measured its magnetic properties from liquid helium temperature to Neel temperature and obtained the latter 237 K [17]. Similar studies have been reported by Venugopal Rao et al on polycrystalline powder prepared by citric acid route [19]. Liu et al have studied the effect of $\mathrm{Ca}^{2+}$ ion doping on the electrical properties of $\mathrm{PrCrO}_{3}$ and proved the applicability of the compound for a superior solid oxide fuel cell (SOFC) [32]. Prasad et al reported the relaxor-like giant permittivity in $\mathrm{PrCrO}_{3}$ and attributed it to the grain boundary effects [18]. From this, we conclude that only a few attempts have been made to tailor the structural and magnetic properties of $\mathrm{PrCrO}_{3}$ chromite through site engineering which is commonly known as substitution or doping.

Recently, another interesting phenomenon called magnetocaloric effect (MCE) has been reported in chromites. Magnetic refrigeration (MR) measured through the indirect measurement of MCE in the cryogenic temperature range has been carried out systematically and carefully. In fact, giant MCE has been observed near the magnetic phase transitions in rare-earth chromites and multiferroic manganites [26, 31, 33, 34].

In this paper, we have investigated the influence of $\mathrm{Mn}$ ion doping on the structural magnetic, magnetocaloric and specific heat properties of $\mathrm{PrCrO}_{3}$ compound. To the best of our knowledge, this is for the first time that such studies are being reported on $\mathrm{Mn}$ doped $\mathrm{PrCrO}_{3}$ orthochromites.

\section{Experimental details}

Polycrystalline samples of $\mathrm{PrCr}_{0.85} \mathrm{Mn}_{0.15} \mathrm{O}_{3}$ (PCMO) were prepared by the solid state reaction method in ambient conditions by taking high purity $\operatorname{Pr}_{6} \mathrm{O}_{11}, \mathrm{MnO}_{2}$ and $\mathrm{Cr}_{2} \mathrm{O}_{3}$ as starting materials (all chemicals from Sigma Aldrich). The mixture was ground thoroughly and first calcined for $24 \mathrm{~h}$ at $600{ }^{\circ} \mathrm{C}$ followed by crushing and second calcination at $900{ }^{\circ} \mathrm{C}$ for $12 \mathrm{~h}$. After second calcination the powder was pulverized and pelletized in the form of uniform and compact pellets, which were sintered at $1350{ }^{\circ} \mathrm{C}$ for $24 \mathrm{~h}$ in air. The phase identification was carried out by x-ray diffractometer (PANalyticalEmpyrean) using $\mathrm{Cu} \mathrm{K}$ radiation with wavelength $1.540 \AA$ at a scanning rate of $0.02^{\circ}$ from $20^{\circ} \leqslant \theta \leqslant 80^{\circ}$. The magnetic measurements were observed by vibrating sample magnetometer (VSM) between $4 \mathrm{~K}-300 \mathrm{~K}$. The isotherms $M(H)$ measurements were measured between 0 and $90 \mathrm{kOe}$ from $2 \mathrm{~K}$ to $102 \mathrm{~K}$.

\section{Result and discussions}

\subsection{Structural analysis}

Figure 1(a) shows the x-ray diffraction patterns of sintered PCMO sample powder along with the Rietveld refinement using Fullprof Suite software [35]. The material is G-type $(a>b / \sqrt{ } 2>c)$ orthorhombic perovskite (Pnma space group) single phase in nature with lattice parameters $a=5.491 \pm$ $0.002 \AA, b=7.7167 \pm 0.004 \AA$ In place of this symbol please use the symbol like after 1.540 in the 11 th line in the Experimental details. Please use that symbol everywhere in paper in place this symbol and $c=5.4525 \pm 0.003 \AA$. The lattice volume of PCMO unit cell is larger than that reported for pristine $\mathrm{PrCrO}_{3}$ sample [36] which can be attributed to the larger size of $\mathrm{Mn}^{+3}(0.645 \AA)$ ion when compared with $\mathrm{Cr}^{+3}$ $(0.615 \AA)$ ion. The results of the refinement are summarized in table 1. 
Table 1. Structural parameters and atomic positions for PCMO powder at room temperature.

$R_{\mathrm{p}}=11.9 \%, R_{\mathrm{wp}}=9.45 \%, R_{\exp }=6.33 \%$, density $=7.294 \mathrm{~g} \mathrm{~cm}^{-3}$, $S=(7.03 \pm 0.4) \times 10^{-3}, \mathrm{~B}-\mathrm{O} 1=1.976(\AA), \mathrm{Pr}-\mathrm{O} 1=2.3475(\AA)$ $\mathrm{B}-\mathrm{O} 1-\mathrm{B}=154.806\left(^{\circ}\right)$, and $\mathrm{B}-\mathrm{O} 2-\mathrm{B}=153.814\left(^{\circ}\right)$

\begin{tabular}{lllll}
\hline Atom & $x$ & $y$ & $z$ & Occ \\
\hline Pr: $4 c(x, 0.25, z)$ & 0.03664 & 0.25 & -0.00536 & 1.0128 \\
Cr: $4 b(0,0,0.5)$ & 0 & 0 & 0.5 & 0.8461 \\
Mn: $4 b(0,0,0.5)$ & 0 & 0 & 0.5 & 0.1516 \\
O1: $4 c(x, 0.25, z)$ & 0.48616 & 0.25 & 0.07783 & 0.9984 \\
O2: $8 d(x, y, z)$ & 0.29633 & 0.04511 & -0.27708 & 2.8443 \\
\hline
\end{tabular}

The spin-lattice interaction dictates the spin configuration and the tilting of $\mathrm{BO}_{6}$ octahedra (inset figure 1(a) made by VESTA software [37]) for perovskite cell, where $\mathrm{B}$ is $\mathrm{Cr} / \mathrm{Mn}$ ion. In $\mathrm{BO}_{6}$ octahedron, $3 d$ degenerate orbitals split into two states due to crystal field splitting: one with the lower energy state and the other is higher energy state named as $t_{2 \mathrm{~g}}$ and $e_{\mathrm{g}}$, respectively [38]. Further hyperfine splitting of $e_{\mathrm{g}}$ orbitals known as Jahn-Teller splitting is possible if these orbitals contain odd number of electrons. Such type of splitting leads to the spin-lattice coupling and ensuing tilting of $\mathrm{BO}_{6}$ octahedra. The sizable mismatch between $\mathrm{R}-\mathrm{O}$ and $\mathrm{B}-\mathrm{O}$ bond lengths in the Goldschmidt tolerance factor $t \equiv(\mathrm{R}-\mathrm{O}) / \sqrt{2} \times(\mathrm{B}-\mathrm{O})$ results in $t<1$ and significant cooperative rotations of the $\mathrm{BO}_{6}$ octahedra (the average ionic radii $\left(R_{\mathrm{b}, \mathrm{avg}}\right)$ of the $\mathrm{B}$-site ions is calculated by $\sqrt{0.15 \times r_{\mathrm{Mn}}^{2}+0.85 \times r_{\mathrm{Cr}}^{2}}$, i.e. $R_{\mathrm{b}, \mathrm{avg}}=0.6196 \AA$, considering the atomic number ratio and ionic radii of $\mathrm{Cr}^{3+}$ $(0.615 \AA)$ and $\mathrm{Mn}^{3+}(0.645 \AA)$ ions $)$. This leads to the bending of the $\mathrm{B}-\mathrm{O}-\mathrm{B}$ angle and hence the distortion in the structure is witnessed. The orthorhombic distortion of the unit cell from the ideal cubic structure, defined by the orthorhombic strain factor $S=2(a-c) /(a+c)$ [39], was calculated and summarized in table 1 as well. It should also be mentioned here that with the addition of $\mathrm{Mn}$ at the $\mathrm{Cr}$-site in $\mathrm{PCMO}$, bond angles (in-plane B-O1-B and out-of-plane B-O2-B) increase which can be understood in terms of the bigger ionic size of $\mathrm{Mn}^{3+}$ than $\mathrm{Cr}^{3+}$ ion that provides a lesser space for the bond to tilt. All these results further indicate the incorporation of $\mathrm{Mn}$ ion at $\mathrm{Cr}$-sites in PCMO compound.

Further, Williamson-Hall relation was utilized to calculate the crystallite size [40]:

$$
\beta_{\text {total }}=\frac{k \lambda}{d_{\mathrm{XRD}} \cos \theta}+4 \eta \tan \theta
$$

where $\beta_{\text {total }}$ is the full width at half-maximum (FWHM) of the XRD peaks. It includes both instrumental and sample dependent broadening effects. $k$ is the Debye-Scherrer constant ( 0.94 for spherical crystallites), $\lambda$ is the incident $\mathrm{x}$-ray wavelength (1.540 $\AA$ ), $\theta$ is the diffraction angle, and $\eta$ is the microstrain parameter. Figure 1(b) shows a plot of $\beta_{\text {total }} \cos \theta / k \lambda$ and $4 \sin \theta / k \lambda$, the estimated values of the average crystallite size $d_{\mathrm{XRD}}$ and the microstrain parameter $\eta$ are $125 \pm 14 \mathrm{~nm}$ and $(2.02 \pm 0.68) \times 10^{-3}$, respectively. The order of microstrain value fairly matches with the orthorhombic distortion $S$ calculated before.

\subsection{Magnetic properties}

Figure 2(a) demonstrates the magnetization versus temperature variation under 200 Oe applied field in the field cooled (FC) mode. One can notice that magnetization is positive throughout the measurement range and increases with decreasing temperature. The maximum value of magnetization for the present compound is almost eight times higher when compared with that of the undoped $\mathrm{PrCrO}_{3}$ material's magnetization even when it was measured under $1 \mathrm{~T}$ magnetic field [19]. This comparison attests the influence of Mn doping in enhancing the magnetic properties of chromites at low temperatures. In order to identify any magnetic transition, the derivative of magnetization with temperature i.e. $\mathrm{d} M / \mathrm{d} T$ was plotted against temperature and the results are shown in figure 2(b) wherefrom two transitions at $T_{\mathrm{N} 1} \sim 197 \mathrm{~K}$ (from paramagnetic to antiferromagnetic state and usually defined as Neel temperature) and $T_{\mathrm{N} 2} \sim 17 \mathrm{~K}$ can be discerned. It is worth mentioning here that the Neel temperature of the undoped PCO sample is $237 \mathrm{~K}$ [17], whereas in the present case it decreases to $197 \mathrm{~K}$ with $15 \%$ of Mn doping. This can be understood in terms of the appearance of double exchange interaction between $\mathrm{Mn}^{3+}$ and $\mathrm{Cr}^{3+}$ ions via $\mathrm{O}^{2-}$ leading to decrease in antiferromagnetic superexchange interaction. Thereby, $T_{\mathrm{N} 1}$ can be expected to decrease with Mn-doping. Further, $T_{\mathrm{N} 2}$ in figure 2(b) is assigned as a spin reorientation temperature due to ordering of $\mathrm{Pr}^{3+}-\mathrm{Pr}^{3+}$ ions spins $\left(4 f^{2}\right.$; effective paramagnetic moment $\mu_{\text {eff }}=3.58 \mu_{\mathrm{B}}$ ). It is also argued that there is a spin structure change from the high temperature canted $\Gamma_{4}$ to collinear $\Gamma_{2}$ at $T_{\mathrm{N} 2}$. Figure 2(b) also depicts the inverse of susceptibility against temperature and it indicates that the high temperature paramagnetic region satisfies the Curie-Weiss behaviour $\left(\chi^{-1}=\frac{\mathrm{C}}{T-\theta_{\mathrm{CW}}}\right)$. The calculated effective magnetic moment was $7.45 \mu_{\mathrm{B}}$ (from the Curie constant $\mathrm{C}$ ) along with Curie temperature $\left(\theta_{\mathrm{CW}}\right)$ of $-184 \mathrm{~K}$, which is indicative for antiferromagnetic interactions. However, orthochromites like the present one possess distorted orthorhombic structure and the $\mathrm{Cr}-\mathrm{O}-\mathrm{Cr}$ bond angle $\theta$ deviates from $180^{\circ}$. Consequently, it results in the imperfect superexchange interaction and the ensuing CAFM (ferromagnetic + antiferromagnetic) behaviour below the Neel temperature. On comparing effective magnetic moment and Curie temperature values for the present PCMO compound with undoped PCO one [19] (4.87 $\mu_{\mathrm{B}}$ and $-158 \mathrm{~K}$ ), we can clearly distinguish the contribution of manganese ions in magnetic behavior.

In order to confirm the coexistence of ferromagnetic and antiferromagnetic phases below the Neel temperature, the isothermal magnetization $M(H)$ loops at several characteristic temperatures $2,50,100,150,200$ and $300 \mathrm{~K}$ were acquired in zero field mode (ZFC) and the graphs are shown in figure 2(c). The loops which are symmetric about the field axis exhibit hysteresis but without any saturation. The magnetization increases linearly for all the isothermal loops in the region of larger applied magnetic field. Such loops are also attributed to the coexistence of AFM (high field) and weak FM (low field) states. Several factors contribute to the observation of combined ferromagnetic and antiferromagnetic behaviors below the Néel temperature for the present compound. 

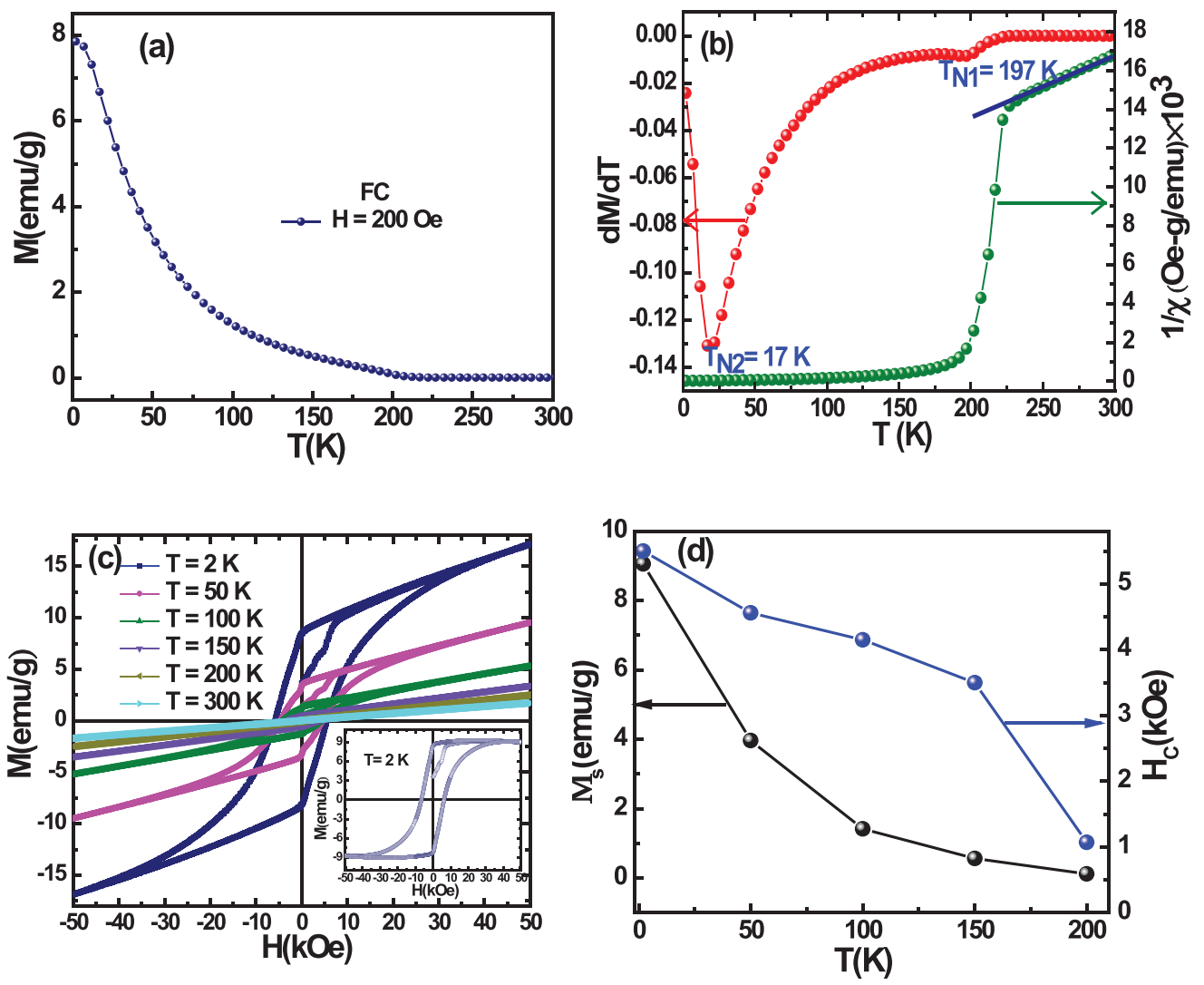

Figure 2. (a) $M(T)$ measured at $H=200 \mathrm{Oe}$ in $\mathrm{FC}$ mode. (b) $\chi^{-1}(T)$ calculated at $H=200 \mathrm{Oe}$, along with $\mathrm{d} M / \mathrm{d} T$ graph. (c) Isothermal $M(H)$ curves at temperatures 2, 50, 100, 150, 200 and $300 \mathrm{~K}$ (the inset is weak FM component versus magnetic field at $2 \mathrm{~K}$ ), (d) the temperature dependence of the saturation magnetization and the coercivity $H_{\mathrm{c}}$ of the weak FM component.
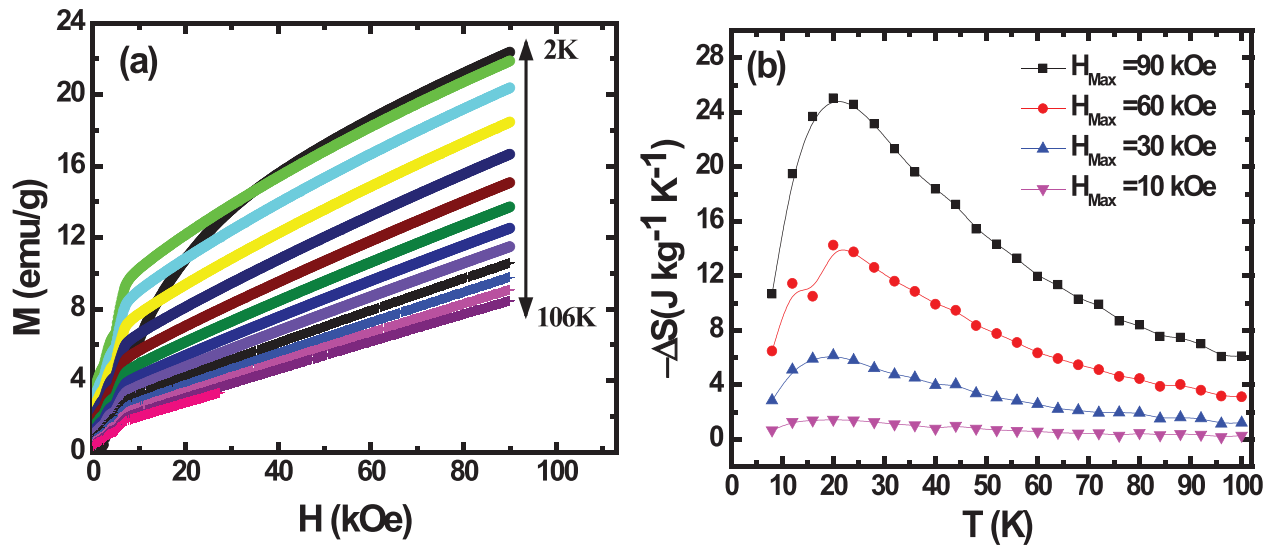

Figure 3. (a) Isothermal magnetization curves, (b) magnetic entropy change versus temperature at different applied magnetic field values.

For example, below $T_{\mathrm{N} 1}$, due to Dzialoshinski-Moriya type antisymmetric exchange interaction, $\mathrm{Cr}^{3+}$ ions are in the canted state resulting in the presence of ferromagnetic and antiferromagnetic states [41, 42]. Moreover, the interaction between $\mathrm{Mn}^{3+}\left(t_{2 \mathrm{~g}}^{3} e_{\mathrm{g}}^{1}\right)$ and $\mathrm{Cr}^{3+}\left(t_{2 \mathrm{~g}}^{3} e_{\mathrm{g}}^{0}\right)$ ions via $\mathrm{O}^{2-}$ would be ferromagnetic as $\mathrm{Mn}$ doping results in the decrease of Néel temperature [43-46]. As the temperature decreases further, $f$-orbitals in $\mathrm{Pr}^{3+}$ ions and $p$-orbitals of oxygen also start interacting and well below the Néel temperature, the $\mathrm{Pr}^{3+}-\mathrm{O}^{2-}$ superexchange interaction also increases, resulting into the onset of $\mathrm{Cr}^{3+}-\mathrm{O}^{2-}-\mathrm{Pr}^{3+}$ interaction. This interaction is ferromagnetic as here the empty $e_{\mathrm{g}}$ orbital of $\mathrm{Cr}^{3+}$ ions overlaps with one end of oxygen $p$-orbital and the other end of the same overlaps with a partially filled $f$-orbital of $\operatorname{Pr}^{3+}$ ion. This weak ferromagnetic superexchange coupling gives rise to opening up of hysteresis loop below $197 \mathrm{~K}$ (shown in figure 2(a)) with decreasing temperature and the remnant magnetization also increases [25]. The net magnetization is thus given by the relation $M(H)=\chi_{\mathrm{AF}} H+M_{\mathrm{s}}$, where $\chi_{\mathrm{AF}} H$ is the antiferromagnetic contribution and $M_{\mathrm{s}}$ is the saturation magnetization of the weak ferromagnetism [47]. The value of $M_{\mathrm{S}}$ can be obtained by subtracting the antiferromagnetic 
Table 2. Magnetic entropy change and relative cooling power at various applied magnetic field.

\begin{tabular}{lll}
\hline$H_{\text {Max }}(\mathrm{kOe})$ & $-\Delta S_{\mathrm{Max}}\left(\mathrm{J} \mathrm{kg}^{-1} \mathrm{~K}^{-1}\right)$ & $\mathrm{RCP}\left(\mathrm{J} \mathrm{kg}^{-1}\right)$ \\
\hline 10 & 1.45 & 34.669 \\
30 & 6.16 & 153.076 \\
50 & 11.44 & 422.136 \\
60 & 14.25 & 526.53 \\
90 & 25.04 & 708.231 \\
\hline
\end{tabular}

contribution from the total magnetization. Inset of figure 2(c) shows the FM contribution for PCMO with the coercive field $H_{\mathrm{c}}=5.5 \mathrm{kOe}$, and saturation magnetization of weak FM component is $M_{\mathrm{s}}=9.06 \mathrm{emu} \mathrm{\textrm {g } ^ { - 1 }}$ at $T=2 \mathrm{~K}$. The values of the $M_{\mathrm{s}}$ and $H_{\mathrm{c}}$ decrease with increasing temperature up to the Neel temperature (figure 2(d)), and thereafter the hysteresis loops apparently disappears, indicating the phase transition to paramagnetic state.

\subsection{Magnetocaloric measurements}

In order to further examine the performance of PCMO compound for MR, $M(H)$ data was acquired by varying the magnetic field from 0 to $90 \mathrm{kOe}$. The data were acquired between 2 to $106 \mathrm{~K}$ temperature range with an interval of $\Delta T=4 \mathrm{~K}$. The results are plotted in figure 3(a) where the data has been shown from 2 to $106 \mathrm{~K}$ at a temperature difference of $8 \mathrm{~K}$ for the sake of clarity. The change of magnetic entropy $(\Delta S)$ caused by MCE is given by [48]:

$$
\Delta S(T)_{\Delta H}=\int_{H_{\mathrm{I}}}^{H_{\mathrm{F}}}\left(\frac{\partial M(T, H)}{\partial T}\right)_{H} \mathrm{~d} H
$$

Generally, for $\Delta S$ calculation from a series of $M-H$ curves, numerical integration of the above Equation by trapezoidal rule results [49]:

$$
\Delta S\left(T_{\mathrm{av}}\right)_{\Delta H}=\frac{\delta H}{2 \delta T}\left(\delta M_{1}+2 \sum_{i=2}^{n-1} \delta M_{i}+\delta M_{n}\right),
$$

where the average temperature $T_{\mathrm{av}}\left[=\left(T_{j}+T_{j+1}\right) / 2\right]$ from the two magnetization isotherms measured at $T_{j}$ and $T_{j+1}$ in a magnetic field changing by $\Delta H=H_{\mathrm{F}}-H_{\mathrm{I}}$ at a constant step $\delta H$. While $\delta T=T_{j+1}-T_{j}$ is the temperature difference between the two isotherms, $n$ is the number of points measured for each of the two isotherms with the magnetic field changing from $H_{1}=H_{\mathrm{I}}$ to $H_{n}=H_{\mathrm{F}}$ at $\delta H=\Delta H /(n-1)$. Further $\delta M_{i}=\left[M\left(T_{j+1}\right)_{i}-M\left(T_{j}\right)_{i}\right]$ is the difference in the magnetization at $T_{j+1}$ and $T_{j}$ for each magnetic-field step from 1 to $n$. It is worthwhile mentioning here that the sign of $-\Delta S$ provides information about the magnetic transition [50]. For a ferromagnetic (FM) transition, a positive value is obtained whereas an antiferromagnetic ordering displays a negative value due to an orientation disorder of the magnetic sublattices. Therefore, it is customary to plot values of $-\Delta S$ against $T$ for different $H$ values. The obtained results for the present PCMO compound are depicted in figure 3(b). It is clear that $-\Delta S$ is positive in the whole temperature measurement range with maximum value observed near $T_{\mathrm{N} 2}$. It suggests the peak due to FM

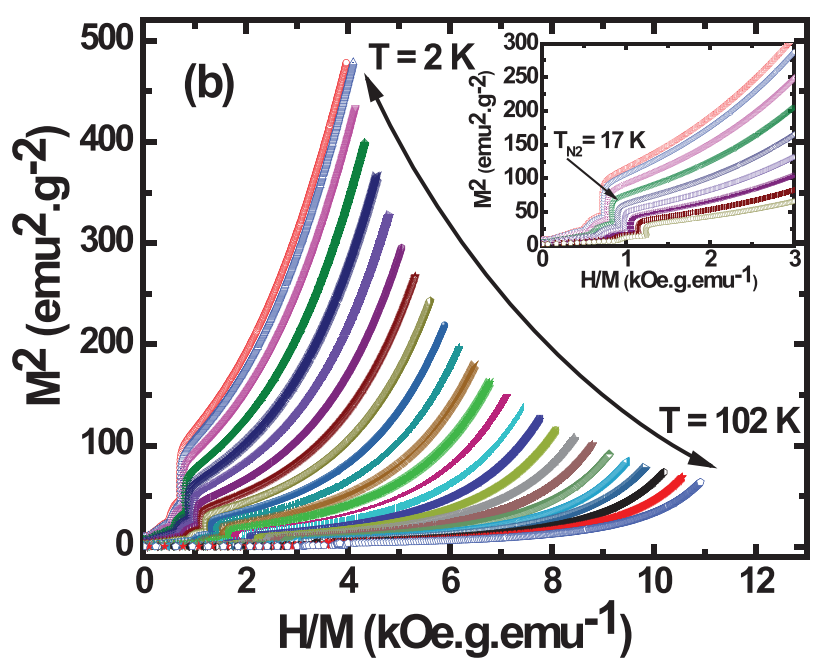

Figure 4. Arrott's plot for PCMO compound. Inset shows the zoomed view.

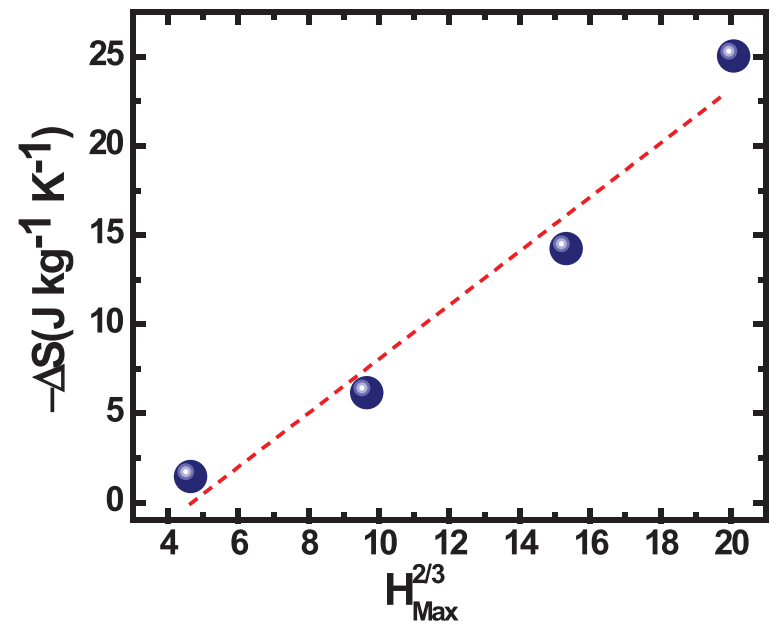

Figure 5. Magnetic entropy variation (maximum) versus $H^{2 / 3}$.

ordering at $T_{\mathrm{N} 2}$. Further, $-\Delta S$ value is $25.04 \mathrm{~J} \mathrm{~kg}^{-1} \mathrm{~K}^{-1}$ at $90 \mathrm{kOe}$. This value is two times higher than the one observed recently by our group in $\mathrm{Mn}$ doped $\mathrm{SmCrO}_{3}$ compound [51]. The difference in the two values could be attributed to the higher magnetic moment associated with $\operatorname{Pr}^{3+}$ ions $\left(3.58 \mu_{\mathrm{B}}\right)$ when compared with that of $\mathrm{Sm}^{3+}$ ions $\left(0.84 \mu_{\mathrm{B}}\right)$. The magnetic entropy variation values for our PCMO compound are superior to (even at smaller applied magnetic field) the results reported on polycrystalline chromites and manganites in the temperature range $10-20 \mathrm{~K}[6,31,34]$. For example, Yoshii [6] observed $-\Delta S$ value is $\sim 4.6 \mathrm{~J} \mathrm{~kg}^{-1} \mathrm{~K}^{-1}$ in the $10-20 \mathrm{~K}$ temperature range under $5 \mathrm{~T}$ applied field whereas it is 11.44 $\mathrm{J} \mathrm{kg}^{-1} \mathrm{~K}^{-1}$ for the compound investigated here (table 2). Yin et al [31] have reported $-\Delta S$ values 7.2 (at 20K), 6.83 (at $20 \mathrm{~K}$ ), $13.08 \mathrm{~J} \mathrm{~kg}^{-1} \mathrm{~K}$ (at $5 \mathrm{~K}$ ) for $\mathrm{HoCrO}_{3}, \mathrm{HoCr}_{0.7} \mathrm{Fe}_{0.3} \mathrm{O}_{3}$, and $\mathrm{DyCr}_{0.7} \mathrm{Fe}_{0.3} \mathrm{O}_{3}$ samples, respectively under $7 \mathrm{~T}$ applied magnetic field. Again looking at the values shown in table 2 we notice better value even at $6 \mathrm{~T}$ magnetic field. Similarly, the present results are better than described by Shao et al on Ho doped $\mathrm{DyMnO}_{3}$ manganites [34]. Further, our $-\Delta S$ values are slightly inferior to that obtained on $\mathrm{GdCrO}_{3}$ single crystals, however, our sample is polycrystalline in nature: easy 

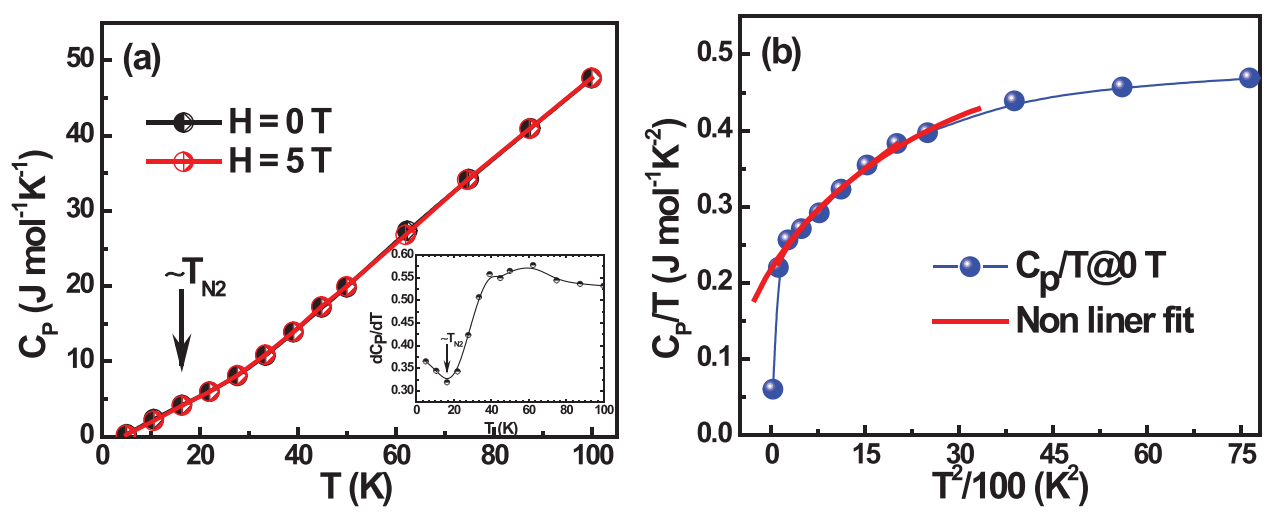

Figure 6. (a) Thermal evolution of heat capacity at zero and $5 \mathrm{~T}$ fields, (b) experimental $C_{\mathrm{p}}$ data is fitted according to the equation $C_{\mathrm{p}}=\gamma T+\beta T^{3}+\delta T^{3 / 2}$ at low temperature region in zero field condition.

to fabricate and cost effective when compared with single crystals.

In the present case, it is apparent that the rotation of $\operatorname{Pr}^{3+}$ ions with applied filed and ordering between them brings positive value corresponding to a FM state in the $T_{\mathrm{N} 2}$ region. It is also envisaged that the alignment of $\mathrm{Mn}^{3+}$ ions along the applied field direction contributes in the enhancement of $-\Delta S$ values. The interaction among the $3 d$ electrons of $\mathrm{Cr}$ and $\mathrm{Mn}$ ions with $6 f$ electrons of Pr ion also leads to enhancement of magnetic entropy change values. We further calculated the relative cooling power (RCP) or refrigerant capacity by the following expression:

$$
\mathrm{RCP}=\left|\Delta S^{\max }\right| \times \Delta T_{\mathrm{FWHM}},
$$

where $\Delta T_{\mathrm{FWHM}}$ is the full width at half maximum of the temperature dependent magnetic entropy change. RCP value is $\sim 371 \mathrm{~J} \mathrm{~kg}^{-1}$ at the maximum field of $90 \mathrm{kOe}$. The $R C P$ values at different fields (table 2) are quite high making the present compound one of the promising candidates for replacement of costlier, environment degrading, gas refrigerant materials in the liquid helium range.

Further the prediction about the order parameter of the magnetic transition could be made with the help of Arrott plot [52] between $H / M$ versus $M^{2}$. The first order phase transition yields negative slope whereas it is positive for second order. In case of PCMO compound, the slope of Arrott plot was found to be positive below $T_{\mathrm{N} 2}$, indicating the transition is of second order. The positive slope was observed above $T_{\mathrm{N} 2}$ signifying that the magnetic transition nature is of second order (figure 3(b) and its inset shows a zoomed view). Thus PCMO compound exhibits large magnetic entropy change and RCP values with second order phase transition near the spin reorientation temperature.

For the materials undergoing a second-order transition, $-\Delta S^{\max }$ was predicted to develop as $H^{2 / 3}$ but later it was found that besides a term in $H^{2 / 3},-\Delta S^{\max }$ contains an extra term independent of $H$ [53]. This was explained on the basis of Landau's theory of second order phase transitions. The obtained expression was [53]:

$$
-\Delta S^{\max }=\alpha\left(\frac{H}{4 b}\right)^{2 / 3}-\frac{\alpha^{2}}{18 b} \Delta T
$$

$\Delta T$ is the width of the distribution of transition temperatures around the mean point, $\alpha$ and $b$ are positive quantities independent of $T$ or $H$ that enter in the standard Landau expansion. Figure 5 shows as $-\Delta S^{\max } \propto H^{2 / 3}$ signalling that an approach based on mean field theory only is sufficient to explain the magnetic behaviour for high fields for the present PCMO sample.

\subsection{Heat capacity}

Since specific heat capacity $\left(C_{\mathrm{P}}\right)$ measurement is sensitive to the phase transition, therefore, to get further insight into the transition nature at $T_{\mathrm{N} 2}$, we measured $C_{\mathrm{P}}$ versus temperature without and with the application of $5 \mathrm{~T}$ magnetic field. The results are shown in figure 6 . Though there is no anomaly apparent near $T_{\mathrm{N} 2}$, on plotting the derivative of specific heat with temperature i.e. $\mathrm{d} C_{\mathrm{P}} / \mathrm{d} T$, a clear dip is observed at $T_{\mathrm{N} 2}$ (inset to figure 6) which is a signature of the second order phase transition in the present studied sample. The heat capacity or specific heat $\left(C_{\mathrm{P}}\right)$ of a magnetic material is given by the equation [54]:

$$
C_{\mathrm{p}}=\gamma T+\beta T^{3}+\delta T^{3 / 2}
$$

where, $\gamma T$ describes the terms related to electronic contribution, $\beta T^{3}$ term gives the contribution from the lattice and $\delta T^{3 / 2}$ gives the magnetic contribution to $C_{\mathrm{P}}$. Further, in the specific heat measurements large hyperfine interaction between the electronic and nuclear spins of magnetic rareearth ion (in this case $\mathrm{Pr}^{3+}$ ) commonly leads to a nuclear Schottky anomaly with a maximum at $\approx 0.3 \mathrm{~K}[55,56]$. Since, in the present study specific heat measurement ranges from 5 to $100 \mathrm{~K}$, therefore, we excluded the hyperfine contribution to the specific heat. The fitting of equation (6) to the data is shown in figure 6(b) and the values of the parameters obtained from the fitting are: $\gamma=0.186 \mathrm{~J} \cdot \mathrm{K}^{-2} \mathrm{~mole}^{-1}, \beta=2.28 \times 10^{-5}$ $\mathrm{J} \cdot \mathrm{K}^{-4}$ mole $^{-1}$ and $\delta=0.0034 \mathrm{~J}$ mole ${ }^{-1} \mathrm{~K}^{-3 / 2}$. From this fit it is clear that magnetic contribution to the total heat capacity is very small as compared to the electronic contribution, therefore, magnetic contribution (which in fact is smaller as we proved above) is masked in figure 6(a). Further, the electronic contribution, $\gamma$ is related to the density of states (DOS) at the Fermi level: $\gamma=\pi^{2} k_{\beta}^{2} N\left(E_{\mathrm{F}}\right) / 3$, where $N\left(E_{\mathrm{F}}\right)$ is the DOS. 
Using $\gamma=0.186 \mathrm{~J} \cdot \mathrm{K}^{-2}$ mole $^{-1}$ yielded a $N\left(E_{\mathrm{F}}\right)=3.2 \times 10^{25}$ $\mathrm{eV}^{-1} \mathrm{~cm}^{-3}$ which is in agreement with the results reported on manganites [54, 57]. The lattice contribution, $\beta$, is related to the Debye temperature $\left(\theta_{\mathrm{D}}\right)$ through the relation $\beta=234 N k_{\beta} / \theta_{\mathrm{D}}^{3}$, where $N$ is the number of ions per mole. The calculated value of $\theta_{\mathrm{D}}$ was $440 \mathrm{~K}$ which is comparable to the $\mathrm{Nd}_{1-x} \mathrm{La}_{x} \mathrm{CrO}_{3}$ system [58]. The overall magnitude of $\theta_{\mathrm{D}}$ is $380-600 \mathrm{~K}$ for $\mathrm{ABO}_{3}$ type perovskites [57, 59]. Since, heat capacity data on undoped $\mathrm{PrCrO}_{3}$ compound is not available in the literature, therefore, it was not possible for us to bring out the difference caused by Mn doping in thermal parameters.

Finally, we could observe unprecedented high value of MCE in PMCO sample near the $\mathrm{T}_{\mathrm{N} 2}$ temperature along with the second order phase transition.

\section{Conclusions}

The impact of Mn doping on the structural, magnetic, magnetocaloric and specific heat properties of PCMO chromites has been investigated. Even with 15\% Mn doping, the crystal structure of the compound remained orthorhombic structure with Pnma space group, however, the lattice volume was found to increase when compared to that of pristine (undoped) $\mathrm{PrCrO}_{3}$ sample. Neel transition temperature decreased with doping of Mn and the magnetization was almost eight times higher than reported for undoped sample. MCE measured through the magnetic entropy change and RCP showed higher values than reported for other polycrystalline chromites and manganites. The Debye temperature and the DOS at Fermi level were also calculated. The material exhibited second order magnetic phase transition between 10-20 K.

\section{Acknowledgments}

The author SK would like to thank University Grant Commission, New Delhi for providing Rajiv Gandhi National Fellowship (RGNF) whereas IC acknowledges the financial support from FCT, Portugal through SFRH/BPD/81032/2011. The authors would also like to thank Council of Scientific and Industrial Research networking projects SURE-CSC0132 and INTELCOAT-CSC0114 for partially supporting this work. ALK acknowledges CICECO-Aveiro Institute of Materials (Ref FCT UID/CTM/50011/2013) financed by national funds through the FCT/MEC and, when applicable, co-financed by FEDER under the PT2020 Partnership Agreement.

\section{References}

[1] Yusuf S M, Kumar A and Yakhmi J V 2009 Temperature and magnetic field controlled magnetic pole reversal in a molecular magnetic compound Appl. Phys. Lett. 95182506

[2] Prejbeanu I L, Kerekes M, Sousa R C, Sibuet H, Redon O, Dieny B and Nozières J P 2007 Thermally assisted MRAM J. Phys.: Condens. Matter 19165218

[3] Cao Y, Cao S, Ren W, Feng Z, Yuan S, Kang B, Bo Lu and Zhang J 2014 Magnetization switching of rare earth orthochromite $\mathrm{CeCrO}_{3}$ Appl. Phys. Lett. 104232405
[4] Gupta P and Poddar P 2015 Temperature and magnetic field-assisted switching of magnetization and observation of exchange bias in $\mathrm{YbCrO}_{3}$ nanocrystals Inorg. Chem. 549509

[5] Yoshii K and Nakamura A 2000 Reversal of magnetization in $\mathrm{La}_{0.5} \mathrm{Pr}_{0.5} \mathrm{CrO}_{3}$ J. Solid State Chem. 155447

[6] Yoshii K 2012 Magnetization reversal in $\mathrm{TmCrO}_{3}$ Mat. Res. Bull. 473243

[7] Bhadram V S, Rajeswaran B, Sundaresan A and Narayana C 2013 Spin-phonon coupling in multiferroic $\mathrm{RCrO}_{3}(\mathrm{R}-\mathrm{Y}$, Lu, Gd, Eu, Sm): a Raman study Eur. Phys. Lett. 10117008

[8] Gupta P, Bhargava R, Das R and Poddar P 2013 Static and dynamic magnetic properties and effect of surface chemistry on the morphology and crystallinity of $\mathrm{DyCrO}_{3}$ nanoplatelets RSC Adv. 326427

[9] Gupta R, Bhargava P and Poddar P 2015 Colossal increase in negative magnetization, exchange bias and coercivity in samarium chromite due to a strong coupling between $\mathrm{Sm}^{3+}-\mathrm{Cr}^{3+}$ spins sublattices J. Phys. D: Appl. Phys. 48025004

[10] Zhao H J, Íñiguez J, Chen X M and Bellaiche L 2016 Origin of the magnetization and compensation temperature in rare-earth orthoferrites and orthochromates Phys. Rev. B 93044117

[11] Yoshii K, Nakamura Y, Ishii A and Morii Y 2001 Magnetic properties of $\mathrm{La}_{1-x} \mathrm{Pr}_{x} \mathrm{CrO}_{3}$ J. Solid State Chem. 162 84-9

[12] Kumar S, Coondoo I, Rao A, Lu B-H, Kuo Y-K, Kholkin A L and Panwar N 2017 Impact of low level praseodymium substitution on the magnetic properties of $\mathrm{YCrO}_{3}$ orthochromites Physica B $\mathbf{5 1 0}$ 104-8

[13] Singh I, Nigam A K, Landfester K, Munoz-Espi R and Chandra A 2013 Anomalous magnetic behavior below $10 \mathrm{~K}$ in $\mathrm{YCrO}_{3}$ nanoparticles obtained under droplet confinement Appl. Phys. Lett. 103182902

[14] Oliveira G N P, Machado P, Pires A L, Pereira A M, Araújo J P and Lopes A M L 2016 Magnetocaloric effect and refrigerant capacity in polycrystalline $\mathrm{YCrO}_{3}$ J. Phys. Chem. Solids 91182

[15] Zhou J-S, Alonso J A, Muonz A, Fernandez-Diaz M T and Goodenough J B 2011 Magnetic structure of $\mathrm{LaCrO}_{3}$ perovskite under high pressure from in situ neutron diffraction Phys. Rev. Lett. 106057201

[16] Shukla R, Bera A K, Yusuf S M, Deshpande S K, Tyagi A K, Hermes W, Eul M and Pottgen R 2009 Multifunctional nanocrystalline $\mathrm{CeCrO}_{3}$ : antiferromagnetic, relaxor, and optical properties J. Phys. Chem. C 11312663

[17] Gordon J D, Hornrcich R M, Shtrikman S and Wanklyn B M 1976 Magnetization studies in the rare-earth orthochromites. V. $\mathrm{TbCrO}_{3}$ and $\mathrm{PrCrO}_{3}$ Phys. Rev. B 133012

[18] Prasad B V, Narsinga Rao G, Chen J W and Babu D S 2011 Relaxor ferroelectric like giant permittivity in $\mathrm{PrCrO}_{3}$ semiconductor ceramics Mater. Chem. Phys. 126918

[19] Rao B V, Prasad B V, Rao G N, Chou F C and Suresh Babu D 2015 Magnetization Reversal in $\mathrm{PrCrO}_{3}$ Adv. Mater. Res. 108696

[20] Indra A, Dey K, Midya A, Mandal P, Gutowski O, Rütt U, Majumdar S and Giri S 2016 Magnetoelectric coupling and exchange bias effects in multiferroic $\mathrm{NdCrO}_{3}$ J. Phys.: Condens. Matter 28166005

[21] Saha S, Chanda S, Dutta A and Sinha T P 2014 Dielectric relaxation and phonon modes of $\mathrm{NdCrO}_{3}$ nanostructure J. Sol Gel Sci. Tech. 89553

[22] Gupta P and Poddar P 2016 Study of magnetic and thermal properties of $\mathrm{SmCrO}_{3}$ polycrystallites RSC Adv. 682014

[23] Huang S, Zerihun G, Tian Z, Yuan S, Gong G, Yin C and Wang L 2014 Magnetic exchange bias and hightemperature giant dielectric response in $\mathrm{SmCrO}_{3}$ ceramics Ceram. Int. 4013937 
[24] El Amrani M, Zaghrioui M, Ta Phuoc V, Gervais F and Massa N E 2014 Local symmetry breaking and spin-phonon coupling in $\mathrm{SmCrO}_{3}$ orthochromite J. Magn. Magn. Mater. 3611

[25] Jaiswal A, Das R, Vivekanand K, Maity T, Abraham P M, Adyanthaya S and Poddar P 2010 Magnetic and dielectric properties and Raman spectroscopy of $\mathrm{GdCrO}_{3}$ nanoparticles J. Appl. Phys. 107013912

[26] Yin L H, Yang J, Kan X C, Song W H, Dai J M and Sun Y P 2015 Giant magnetocaloric effect and temperature induced magnetization jump in $\mathrm{GdCrO}_{3}$ single crystal J. Appl. Phys 117133901

[27] McDannald A, Kuna L, Seehra M S and Jain M 2015 Magnetic exchange interactions of rare-earth-substituted $\mathrm{DyCrO}_{3}$ bulk powders Phys. Rev. B 91224415

[28] Wang S, Hou C, Yuan L, Qu M, Zou B and Lu D 2016 Hydrothermal preparation of perovskite structures $\mathrm{DyCrO}_{3}$ and $\mathrm{HoCrO}_{3}$ Dalton Trans. 4517593

[29] Su Y, Zhang J, Feng Z, Li L, Li B, Zhou Y, Chen Z and Cao S 2010 Magnetization reversal and $\mathrm{Yb}^{3+} / \mathrm{Cr}^{3+}$ spin ordering at low temperature for perovskite $\mathrm{YbCrO}_{3}$ chromites $J$. Appl. Phys. 108013905

[30] Yin S and Jain M 2016 Enhancement in magnetocaloric properties of holmium chromite by gadolinium substitution J. Appl. Phys. 120043906

[31] Yin S, Sharma V, McDannald A, Reboredo F A and Jain M 2016 Magnetic and magnetocaloric properties of iron substituted holmium chromite and dysprosium chromite RSC Adv. 69475

[32] Liu X, Su W and Lu Z 2003 Study on synthesis of $\mathrm{Pr}_{1-x} \mathrm{Ca}_{x} \mathrm{CrO}_{3}$ and their electrical properties Mater. Chem. Phys. 82327

[33] Mcdannald A, Kuna L and Jain M 2013 Magnetic and magnetocaloric properties of bulk dysprosium chromite J. Appl. Phys. 114113904

[34] Shao M J, Cao S X, Yuan S J, Shang J, Kang B J, Lu B and Zhang J C 2012 Large magnetocaloric effect induced by intrinsic structural transition in $\mathrm{Dy}_{1-x} \mathrm{Ho}_{x} \mathrm{MnO}_{3}$ Appl. Phys. Lett. 100222404

[35] Rodríguez-Carvajal J 1993 Recent advances in magnetic structure determination by neutron powder diffraction Physica B 19255

[36] Lazova S-D, Kovacheva D, Aleksovska S, Marinšek M and Tzvetkov P 2012 Synthesis and structural details of perovskites within the series $\mathrm{PrCo}_{1-x} \mathrm{Cr}_{x} \mathrm{O}_{3}(x=0,0.33$, 0.5, 0.67 and 1) Bulg. Chem. Commun. 4437

[37] Momma K and Izumi F 2011 VESTA 3 for three-dimensional visualization of crystal, volumetric and morphology data J. Appl. Crystallogr. 441272

[38] Ray N and Waghmare U V 2008 Coupling between magnetic ordering and structural instabilities in perovskite biferroics: a first-principles study Phys. Rev. B 77134112

[39] Chan T S, Liu R S, Yang C C, Li W H, Lien Y H, Huang C Y and Lee J F 2007 Chemical size effect on the magnetic and electrical properties in the $\left(\mathrm{Tb}_{1-x} \mathrm{Eu}_{x}\right) \mathrm{MnO}_{3}(0 \leqslant x \leqslant 1.0)$ system J. Phys. Chem. B 1112262

[40] Williamson G K and Hall W H 1953 X-ray line broadening from filed aluminium and wolfram Acta Metall. Mater. 122
[41] Moriya T 1960 Anisotropic superexchange interaction and weak ferromagnetism Phys. Rev. 12091

[42] Dzyaloshinsky I 1958 A thermodynamic theory of 'weak' ferromagnetism of antiferromagnetics J. Phys. Chem. Solids 4241

[43] Yoo Y J, Lee Y P, Park J S, Kang J H, Kim J, Lee B W and Seo M S 2012 Spin-glass behavior of Cr-doped $\mathrm{YMnO}_{3}$ compound J. Appl. Phys. 112013903

[44] Li S Z, Wang T T, Han H Q, Wang X Z, Li H, Liu J and Liu J-M 2012 Modulated multiferroicity of Cr-doped orthorhombic polycrystalline $\mathrm{YMnO}_{3} J$. Phys. D: Appl. Phys. 45055003

[45] Dho J, Kim W S and Hur N H 2002 Reentrant spin glass behavior in Cr-doped perovskite mangnite Phys. Rev. Lett. 89027202

[46] Li C L, Huang S, Li X X, Zhu C M, Zerihun G, Yin C Y, Lu C L and Yuan S L 2017 Negative magnetization induced by $\mathrm{Mn}$ doping in $\mathrm{YCrO}_{3}$ J. Magn. Magn. Mater. 43277

[47] Durán A, Arevalo López A M, Castillo-Martínez E, Garía-Guaderrama M, Moran E, Cruz M P, Fernández F and Alario-Franco M A 2010 Magneto-thermal and dielectric properties of biferroic $\mathrm{YCrO}_{3}$ prepared by combustion synthesis J. Solid State Chem. 1831863

[48] Morrish A H 1965 The Physical Principles of Magnetism (New York: Wiley) ch 3

[49] Pecharsky V K and Gschneidner K A Jr 1999 Magnetocaloric effect from indirect measurements: magnetization and heat capacity J. Appl. Phys. 86565

[50] Phan M H, Frey N A, Angst M, de Groot J, Sales B C, Mandrus D G and Srikanth H 2010 Complex magnetic phases in $\mathrm{LuFe}_{2} \mathrm{O}_{4}$ Solid State Commun. 150341

[51] Kumar S, Coondoo I, Vasundhara M, Patra A K, Kholkin A L and Panwar N 2017 Magnetization reversal behavior and magnetocaloric effect in $\mathrm{SmCr}_{0.85} \mathrm{Mn}_{0.15} \mathrm{O}_{3}$ chromites J. Appl. Phys. 121043907

[52] Arrott A 1957 Criterion for ferromagnetism from observations of magnetic isotherms Phys. Rev. 1081394

[53] Lyubina J, Kuz'min M D, Nenkov K, Gutfleisch O, Richter M, Schlagel D L, Lograsso T A and Gschneidner K A Jr 2011 Magnetic field dependence of the maximum magnetic entropy change Phys. Rev. B 83012403

[54] Pickett W E and Singh D J 1996 Electronic structure and halfmetallic transport in the $\mathrm{La}_{1-x} \mathrm{Ca}_{x} \mathrm{MnO}_{3}$ system Phys. Rev. B 531146

[55] Van Kempen H, Miedema A R and Huiskamp W J 1964 Heat capacities of the metals terbium and holmium below $1^{\circ} \mathrm{K}$ Physica 30229

[56] Kumar C M N, Xiao Y, Nair H S, Voigt J, Schmitz B, Chatterji T, Jalarvo N H and Brückel T 2017 Hyperfine and crystal field interactions in multiferroic $\mathrm{HoCrO}_{3} J$. Phys.: Condens. Matter 28476001

[57] Woodfield B F, Wilson M L and Byers J M 1997 Lowtemperature specific heat of $\mathrm{La}_{1-x} \mathrm{Sr}_{x} \mathrm{MnO}_{3+\delta}$ Phys. Rev. Lett. 783201

[58] Du Y, Xiang Cheng Z, Wang X-L and Dou S X 2010 Structure, magnetic, and thermal properties of $\mathrm{Nd}_{1-x} \mathrm{La}_{x} \mathrm{CrO}_{3}(0 \leqslant x \leqslant 1.0) J$. Appl. Phys. 108093914

[59] Salamon M B and Jaime M 2001 The physics of manganites: structure and transport Rev. Mod. Phys. 73583 